\title{
Modélisation du risque d'évolution métastatique chez les patients supposés avoir une maladie localisée
}

Model-based assessment of the risk of metastatic spreading in patients with localized cancer

Verga F (1, 2), You B (2, 3, 4), Benabdallah A (1), Hubert F (1), Faivre C (5), Mercier C (2, 6), Ciccolini J (5, 6), Barbolosi D (5).

(1) UMR CNRS 6632, Université de Provence, LATP, 39 rue F. Joliot Curie, 13453 Marseille Cedex 13.

(2) EA 3738, Université de Lyon, F-69622 Lyon, France; Université Lyon 1, Faculté de Médecine Lyon Sud, F-69600 Oullins, France.

(3) Oncologie médicale, Centre Hospitalier Lyon Sud, Hospices Civils de Lyon, F-69310, PierreBénite, France

(4) Medical oncology department, Drug development program, Princess Margaret Hospital, Toronto, Canada

(5) UMR MD3, Université de la Méditerranée, Faculté de Pharmacie, Laboratoire de Pharmacocinétique, 27, boulevard Jean Moulin 13385 Marseille Cedex 5

(6) Pôle Oncologie, CHU Timone, APHM Marseille.

Mots-clés : Modélisation, Métastases, Pronostic, Croissance tumorale.

Key-words : Modelling, metastatic disease, pronostic, tumor growth.

Titre courant: Evaluation du risque métastatique par modélisation mathématique.

Running-title : Evaluation of metastatic risk by mathematical modeling. 


\section{Résumé}

La stratégie de traitement lors de la prise en charge d'une tumeur solide repose fondamentalement sur le classement initial entre maladie localisée ou diffuse. Si cette classification dichotomique a le mérite de la simplicité, elle ne permet pas de rendre compte d'une réalité beaucoup plus complexe, à savoir l'existence d'un continuum entre ces deux états. Or il est avéré que certains patients initialement identifiés comme ayant une maladie localisée au terme du bilan d'extension classique, aient en fait une maladie métastatique non-mise en évidence par les techniques usuelles d'imagerie. Ce risque implicitement reconnu justifierait l'usage de thérapies adjuvantes après traitement radical de type exérèse chirurgicale. Un grand nombre de travaux ont déjà été réalisés afin d’identifier des marqueurs pronostiques permettant de préciser ce risque invasif chez des patients dont la maladie était apparemment initialement localisée. Dans ce contexte, notre étude basée sur une modélisation mathématique originale, présente un nouveau marqueur, appelé Index Métastatique, permettant de donner une estimation du nombre et de la taille des métastases en fonction du temps. A terme, cet Index Métastatique pourrait fournir un nouvel outil décisionnel précieux qui permettrait de prédire le risque d'évolution métastatique d’une maladie supposée localisée, et éclairer au mieux le clinicien sur l'intérêt d'un éventuel traitement adjuvant. 


\begin{abstract}
Classification of cancer as localized or metastatic disease remains a mainstay for determining next the best therapeutic strategy to be undertaken at bedside. Although simple, this classification may underestimate the risk of undetectable, early metastatic stages. Many studies have been performed to identify prognostic biomarkers for evaluating the risk of metastatic relapse in patients with apparently localized disease. Here, we have developed an original mathematical model designed to calculate a Metastatic Index. This Metastatic Index should enable to better predict the risk of invasive cancer, even when it does not show with standard imaging techniques. Further validation steps are ongoing, both in tumor-bearing animals and as part of retrospective comparisons with clinical data. This new tool should help the clinician to undertake systemic treatment at the earliest stages of the disease, thus allowing a better efficacy eventually.
\end{abstract}




\section{$\underline{\text { 1- Introduction }}$}

Lors de la prise en charge d'un patient atteint de cancer, les cliniciens ont coutume de systématiquement différencier maladie localisée et maladie métastatique. La première fait l'objet d'un traitement agressif, comprenant souvent une prise en charge radicale telle qu'une chirurgie d'exérèse ou une radiothérapie et un traitement systémique néo-adjuvant/adjuvant, afin d'offrir le maximum de chances de rémission. Dans la seconde situation, un traitement systémique donné seul sera préféré parce que les objectifs de qualité de vie et de prolongation de survie prévalent, exceptions faites de quelques cas de métastases curables ou résécables. Si cette classification binaire (maladie localisée VS. métastatique) offre l'avantage de sa simplicité et reflète dans une certaine mesure les résultats des études pronostiques, elle ne rend sans doute pas compte d'une réalité plus complexe : l'existence d'un probable continuum entre ces deux stades d'extension carcinologiques. En effet, entre la maladie purement limitée au site primitif et la maladie reconnue métastatique, il existe de nombreuses situations dans lesquelles la tumeur pourra être jugée localisée alors qu'elle est déjà diffuse, parce que les moyens diagnostiques disponibles n'ont pas pu mettre en évidence ces lésions (métastases de très petite taille, région du corps non explorée,...). Par ailleurs, la chimiothérapie adjuvante destinée à traiter, après chirurgie, d'éventuels îlots cellulaires cancéreux non identifiés lors du bilan initial d'extension pourrait être considérée comme une reconnaissance implicite de ce continuum. D'autre part, le concept moderne de curabilité des métastases de cancer colorectal ou du testicule recouvre aussi cette notion de lien continu entre maladie limitée curable et maladie diffuse souvent considérée comme incurable. Parce que le choix du traitement dépend essentiellement du staging initial de la maladie, cette sélection réduite à une dichotomie entre deux classes peut paraître comme scientifiquement erronée, tout au moins pour le groupe des maladies localisées. En effet, parmi 
les patients atteints d'un cancer visiblement localisé dont la résection est complète, certains ont un résidu tumoral nul pour lesquels un traitement adjuvant serait superflu, alors que d'autres ayant une maladie métastatique non identifiable devraient impérativement bénéficier d'un tel traitement [1], [2]. Depuis la date $t=0$ d'apparition de la première cellule tumorale à l'origine de la tumeur primitive, s'ensuit une période, dite infraclinique, pendant laquelle la tumeur reste indétectable. La plus petite lésion détectable par imagerie mesure généralement plus de $5 \mathrm{~mm}$. Etant donné qu'une tumeur mesurant $1 \mathrm{~mm}^{3}$ est supposée contenir $10^{6}$ cellules [3], nous avons alors considéré que la plus petite tumeur décelable par imagerie était composée d'au moins $10^{8}$ cellules. Le temps $T_{0}$ a été défini comme étant la date du diagnostic (précisément la durée de temps séparant l'apparition de la première cellule cancéreuse et la date du diagnostic), à laquelle la tumeur primitive est supposée être composée d'au moins $10^{8}$ cellules. Tout cancer débutant peut être considéré comme potentiellement métastatique [1] depuis son origine jusqu'à la date $T_{0}$. Ainsi la tumeur primitive aura pu essaimer sans qu'aucune des métastases ne soit encore visible lors d'un bilan d'extension, car composées de moins $10^{8}$ cellules. Il s'avère essentiel d'estimer le nombre de métastases encore non détectables qui émergeront potentiellement dans l'organisme à une date $T_{1} \geq T_{0}$ c'est-à-dire le nombre de métastases composées d'au moins $10^{8}$ cellules, censées exister à la date $T_{1}$. L'objectif de ce travail est de forger un nouvel index venant compléter les classifications usuelles (TNM, FIGO,...) afin de mieux dépister les patients à haut risque de métastases dans un contexte de bilan d'extension tumoral négatif. Le calcul de cet index, que nous appellerons index métastatique (IM), repose sur une modélisation mathématique que nous avons publiée et décrite in extenso précédemment [4]. 


\section{2 - Matériel et méthode}

\section{2-1 - Rappel et présentation du Modèle}

Les équations et démonstrations mathématiques ayant présidé au développement du modèle ont déjà été présentées dans une publication séparée [4]. Ce modèle décrit au cours du temps l'évolution du nombre et de la taille des différents sites métastatiques développés à partir d'une tumeur primitive donnée. Le modèle a été construit pour donner une estimation du nombre de métastases présentes à l'instant $t$ et qui sont composées d'au moins $p$ cellules [4]. Ce nombre sera par définition l'index métastatique au temps $t$ pour la taille $p$. En particulier en prenant pour taille $10^{8}$ (i.e. $p=10^{8}$ ) le modèle donne donc une estimation du nombre de métastases détectables à la date $t$. Pour la taille 1 (i.e. $p=1$ ) le modèle donne alors une estimation du nombre total de métastases existantes, détectables ou non, à la date $t$. Quand nous parlerons de l’index métastatique à la date $t$ sans préciser la taille il sera sous entendu que c'est pour la taille $10^{8}$, c'est-à-dire pour les métastases détectables. Quand on ne précisera pas la date $t$ il sera sousentendu que c'est à la date du diagnostic, c'est à dire pour $t=T_{0}$. Ainsi quand pour un patient donné nous dirons que son index métastatique est nul, cela signifiera donc qu'à la date du diagnostic le modèle indique que ce patient n'a pas de métastase détectable. L’intérêt d'un tel modèle réside dans sa capacité prédictive c'est-à-dire dans notre cas, sa capacité à estimer si le patient aura des métastases détectables après la date du diagnostic. Par exemple en calculant son index métastatique à la date $\mathrm{t}=T_{0}+3$, le modèle donnera une estimation du nombre de métastases détectables 3 ans après la date du diagnostic (c'est ce que nous appellerons alors l'index métastatique à 3 ans). Le modèle est caractérisé par 4 paramètres que nous avons notés $a, b, m, \alpha$. Les deux premiers paramètres $a, b$ sont liés au développement de la tumeur qui est supposé 
suivre classiquement un modèle de Gompertz. Le paramètre $a$ traduit la vitesse de prolifération, relié au temps de doublement tumoral et le paramètre $b$ reflète le nombre maximal de cellules composant la tumeur. Ce nombre étant généralement compris entre $10^{11}$ et $10^{12}$ cellules, ce qui correspond à une masse respectivement comprise entre $100 \mathrm{~g}$ et $1 \mathrm{~kg}$ [3]. Les deux autres paramètres $m$ et $\alpha$ sont reliés plus spécifiquement au processus métastatique. Nous avons supposé que le nombre de cellules migrantes qui se détachent (par unité de temps) d'une tumeur composée de $x$ cellules vaut $m x^{\alpha}$ (voir FIG.1). Les 4 paramètres $a, b, m, \alpha$ sont spécifiques à chaque patient pour un type de cancer donné et permettent de calculer son index métastatique. Cependant ce modèle n’a pas encore été validé de manière définitive. Nous présenterons ici une validation « in silico » du modèle, basée sur des études de simulation. Les paramètres $a$ et $b$ peuvent être estimés à partir du temps de doublement et de l'observation par imagerie de la tumeur primitive en deux dates successives. En ce qui concerne les deux autres paramètres, rechercher des covariables pourraient permettre de les estimer indirectement. Ces covariables sont évidemment à chercher parmi celles qui sont liées au processus métastatique. On sait ainsi que le développement de métastases est lié à la chute du nombre de certaines molécules d'adhésions telles les cadhérines E [5] ainsi qu’à la néo-angiogénèse tumorale, stimulée par les facteurs de croissance tel le VEGF. Nous pensons que les deux paramètres $m$ et $\alpha$ peuvent être corrélés à ces deux événements, mais cela doit être confirmé par une étude de population ultérieure. 


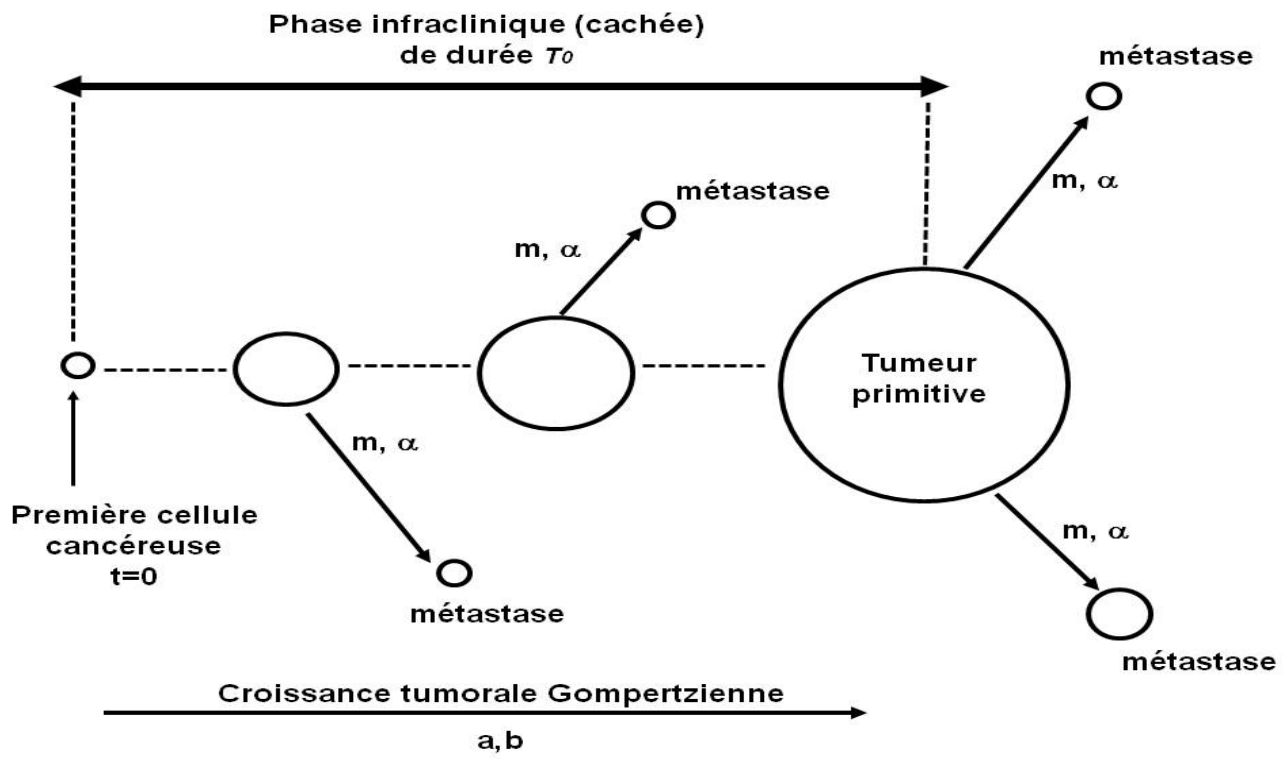

FIG.1 - Schéma du processus tumoral depuis son origine monoclonale jusqu’aux métastases

\section{2-2 - Confrontation des simulations du modèle avec des données cliniques}

Comme exposé précédemment, les 4 paramètres $a, b, m, \alpha$ sont spécifiques à chaque patient pour un type de cancer donné. Une étude de population peut alors permettre de déterminer les valeurs moyennes (valeurs de population) ainsi que la distribution de ces paramètres dans la population des patients. Dans le cadre du cancer du sein nous avons essayé de voir si l'on pouvait donner une distribution pour ces paramètres qui permette ensuite par simulation de retrouver des résultats de la littérature afin de donner une validation (certes encore incomplète) du modèle. Dans une étude rétrospective, impliquant 2648 patientes atteintes de cancer du sein traitées à l'Institut Gustave Roussy entre 1954 et 1972, Koscielny et al. [6] ont déterminé en fonction de la taille initiale de la tumeur la proportion des malades qui ont eu par la suite au moins une métastase. Comme dans Koscielny, nous avons considéré les 8 plages de taille suivantes pour la tumeur primitive : de 1.5 à 2.5 , de 2.5 à $3.5, \ldots$, de 7.5 à $8.5 \mathrm{~cm}$ et plus grandes que $8.5 \mathrm{~cm}$. La distribution 
de $a, b, m, \alpha$ a été choisi comme suit : les deux paramètres $a, b$ ont été en fait fixés. Le paramètre $a$ du modèle Gompertz pour l'évolution de la tumeur a été déterminé pour décrire une tumeur qui aurait un temps de doublement de 2 mois, ce qui correspond au temps de doublement moyen pour une tumeur humaine. Ainsi, on a pris: $a=0.000471$ et $b=10^{12}$. Pour les paramètres $m$ et $\alpha$, on a supposé pour tous les deux une répartition normale dans la population des patientes avec $\mu_{m}=2.5 \times 10^{-8}$ et $\sigma_{m}=10^{-8}$ pour $m$ et $\mu_{\alpha}=0.48$ et $\sigma_{\alpha}=0.3$ pour $\alpha$. Pour chaque plage de taille, nous avons procédé à $N=100$ simulations correspondant intuitivement à 100 patientes. Pour chaque patiente, nous avons d'abord tiré au hasard (de manière uniforme) une taille dans la plage considérée puis une valeur de $m$ et $\alpha$ suivant la distribution décrite ci-dessus. On dispose alors des quatre paramètres $a, b, m, \alpha$ pour la patiente (rappelons que $a$ et $b$ sont fixés avec $a=0.000471$ et $b=10^{12}$ ). Ensuite nous avons calculé la durée $T_{0}$ écoulée depuis la naissance de la première cellule cancéreuse et le moment du diagnostic (en fait cette durée $T_{0}$ ne dépend que $a$ et $b$ donc c'est la même pour toutes les patientes puisque $a$ et $b$ sont fixées) puis nous avons calculé l'index métastatique pour la taille 1 . Ceci étant fait pour les $N=100$ patientes de l'échantillon de simulation, nous avons regardé ensuite le nombre $N_{\text {meta }}$ de patientes pour lesquelles cet index métastatique était supérieur ou égal à 1 (présence d’au moins une métastase). Ce nombre $N_{\text {meta }}$ coïncide donc avec le nombre de patientes qui ont au moins une métastase, décelable ou non, lors du diagnostic. Sans traitement supplémentaire, ces patientes devraient avoir au moins une métastase détectable à une date ultérieure à $T_{0}$. Ainsi, pour chacune des plages de taille, le rapport $\frac{N_{\text {meta }}}{N}$ représente la proportion théorique prévue par le modèle des patientes qui devraient développer secondairement une métastase détectable sans traitement adjuvant. Ces proportions ont été comparées avec celles de Koscielny. 


\section{2-3 - Variations de la valeur des paramètres $m$ et $\alpha$}

Nous avons illustré l'impact de la variabilité des paramètres $m$ et $\alpha$ sur la valeur de l'index métastatique. De la même façon que dans l'exemple précédent nous avons considéré les cas de patientes atteintes de cancer du sein dont les paramètres de croissance Gompertzienne étaient fixés à : $a=0.000471, b=10^{12}$. Ceci conduit alors à une estimation de $T_{0}=2943$ jours (soit une période cachée d'environ 8 ans) afin d'atteindre une masse tumorale composée de $10^{9}$ cellules (plus petite tumeur détectable par palpation).

\section{2-3-1 - Variation du paramètre $m$.}

Nous avons fixé le paramètre $\alpha$ à 0.5 , puis nous avons choisi quatre valeurs de $m$ entre $2.5 \times 10^{-9}$ et $2.5 \times 10^{-6}$ de manière à simuler le cas de quatre patientes, chacune ayant une tumeur primitive constituée de $10^{9}$ cellules et n'ayant aucune métastase visible à la date du diagnostic mais ayant une grande variabilité de leur index métastatique à 3 et 5 ans.

\section{2-3-2- Variation du paramètre $\alpha$}

Le paramètre $m$ a été fixé à $2.5 \times 10^{-7}$ puis nous avons choisi quatre valeurs de $\alpha$ entre 0.2 et 0.8 de manière à simuler à nouveau le cas de quatre patientes n'ayant aucune métastase visible à la date du diagnostic mais ayant une grande variabilité de leur index métastatique à 3 et 5 ans.

\section{2-4- Variation du paramètre $m$ et simulation du possible impact de la chirurgie sur l’angiogenèse.}


Peeters et al. [7] ont suggéré que la chirurgie carcinologique pourrait favoriser une flambée métastatique par le biais du phénomène d'angiogenèse [7]. La tumeur primitive freinerait le développement de métastases en secrétant des substances inhibitrices de l'angiogenèse (angiostatine et endostatine). L'exérèse tumorale supprimerait ce frein et favoriserait donc le risque de diffusion métastatique. Cette flambée serait d'autant plus importante que la tumeur est grosse, d’où l’intérêt de la réduire au préalable par une chimiothérapie néo-adjuvante. Nous avons évalué les capacités de notre modèle à pouvoir reproduire une telle hypothèse clinique, si elle s’avérait recevable. Cet éventuel accroissement brutal du mécanisme d'angiogenèse pourrait être intégré dans notre modèle par une augmentation rapide de la valeur du paramètre $m$. Nous pouvons supooser aue la chirurgie pourrait être une covariable explicative du paramètre $m$ à travers par exemple une relation de la forme $m=u+(v+w T) X$ où $u, v, w$ sont des constantes, $X$ la covariable chirurgie ( $X=1$ si chirurgie et 0 sinon) et $T$ la taille de la tumeur. Sans chirurgie, on aurait $m=u$ et avec chirurgie $m=u+v+w T$.

Dans cette modélisation, l'effet de la chirurgie sur $m$ dépendrait de la taille de la tumeur T. Plus cette taille serait importante plus l'augmentation de $m$ serait importante en accord avec l’hypothèse de Peeters et al. Nous avons illustré ce qui précède en prenant pour modèle trois patientes ayant respectivement une masse tumorale primitive de $1 \mathrm{~g}, 50 \mathrm{~g}$ et $100 \mathrm{~g}$ et donc composées de $10^{9}, 5 \times 10^{10}$ et $10^{11}$ cellules. Pour chacune de ces tumeurs la chirurgie a été considérée comme radicale, c'est à dire avoir ramené le nombre de cellules tumorales idéalement à une dizaine de cellules. Les paramètres de la croissance Gompertzienne étaient fixés à $a=0.000471, b=10^{12}$ tels que définis antérieurement pendant que $\alpha$ était fixé à 0.5. Nous avons supposé que la valeur du paramètre $m$ était $2.5 \times 10^{-8}$ avant chirurgie pour les 3 patientes. 
Comme exposé plus haut, nous avons supposé que l’acte chirurgical produisait une flambée d'autant plus importante que la tumeur est grosse par une majoration croissante de la valeur de $m$. Ainsi les valeurs de $m$ ont été respectivement fixées à $2.5 \times 10^{-7}, 2.5 \times 10^{-6}$ et $2.5 \times 10^{-5}$ après la chirurgie. Nous avons alors calculé pour chaque patiente l'index métastatique à 5 ans et à 10 ans.

\subsection{Variation du paramètre $m$ et traitement par chimiothérapie.}

Le modèle mathématique pour l'index métastatique est également susceptible de prendre en compte l'effet d’une chimiothérapie. Nous nous plaçons ici dans le cadre du cancer du sein métastatique. Nous présentons l'effet d'une chimiothérapie de l'association DocetaxelEpirubicine sur l'évolution de l'index métastatique. Le protocole simulé ici est celui préconisé dans l'étude de Viens et al. [8] qui consiste à effectuer 6 cycles de 21 jours avec perfusion d'une heure de $75 \mathrm{mg} / \mathrm{m}^{2}$ pour le Docetaxel, suivi d'une heure de repos puis d'une perfusion d'un quart d'heure de $100 \mathrm{mg} / \mathrm{m}^{2}$ pour l'Epirubicine, à J1. Les modèles pharmacocinétiques utilisés sont des modèles à trois compartiments pour chacun des deux médicaments, et les valeurs des paramètres pharmacocinétiques sont issues d’un travail préalable [9]. La modélisation mathématique pharmacodynamique traduisant l'effet des médicaments a été détaillée préalablement $[9,10]$. Comme précédemment les paramètres de la croissance Gompertzienne ont été fixés à $a=0.000671, b=10^{12}$ et $\alpha$ a été fixé à 2/3. La masse tumorale initiale a été choisie de $3 \mathrm{~g}$.

Quatre valeur de $m$ ont été tirées aléatoirement et nous avons calculé, dans chacun de ces cas, l'évolution de l'index métastatique 1 an et 2 ans après la fin d'un traitement commun de 6 cycles de chimiothérapie (la durée du traitement $T_{\text {trait }}$ est donc estimée à 126 jours). 


\section{3 - Résultats}

\section{3-1 - Confrontations des simulations du modèle avec des données cliniques}

La Table 1 présente en fonction de la taille de la lésion primitive, les pourcentages de maladies métastatiques rapportées par Koscielny et al. (colonne 3 )et les proportions prédites par le modèle (colonne 2) des patientes susceptibles d'avoir une métastase. La similitude entre les valeurs observées et les prédictions du modèle est confirmée par le calcul du coefficient de corrélation qui est de 0.98 .

\begin{tabular}{|c|c|c|}
\hline \multirow{2}{*}{ Taille de la tumeur primitive } & Pourcentages calculés $\frac{N_{\text {meta }}}{N}$ & Pourcentages observés dans [6] \\
\hline $1-2.5 \mathrm{~cm}$ & $25.5 \%$ & $27 \%$ \\
\hline $2.5-3.5 \mathrm{~cm}$ & $44.25 \%$ & $42 \%$ \\
\hline $3.5-4.5 \mathrm{~cm}$ & $60.5 \%$ & $56.7 \%$ \\
\hline $4.5-5.5 \mathrm{~cm}$ & $68.6 \%$ & $66.5 \%$ \\
\hline $5.5-6.5 \mathrm{~cm}$ & $75.5 \%$ & $72.8 \%$ \\
\hline $6.5-7.5 \mathrm{~cm}$ & $78.25 \%$ & $83.8 \%$ \\
\hline $7.5-8.5 \mathrm{~cm}$ & $83.25 \%$ & $81.3 \%$ \\
\hline$>8.5 \mathrm{~cm}$ & $89.25 \%$ & $92 \%$ \\
\hline
\end{tabular}

Tab. 1-En fonction de la taille de la tumeur primitive, pourcentage des patients ayant au moins une métastase, théorique vs observée.

\section{3-2 - Variations de la valeur des paramètres $m$ et $\alpha$}

Ces résultats montrent que la variabilité du nombre de métastases chez une patiente peut être décrite par une variabilité sur les paramètres $m$ et $\alpha$. Les effets de variations du paramètre 
$m$ sur l'index métastatique à 3 et 5 ans sont présentés dans la Table 2. Alors que toutes les patientes sont supposées avoir une maladie localisée (ce qui est traduit par un index métastatique nul à $T_{0}$ ), celles présentant une faible valeur du paramètre $m$ ne développeraient pas de métastase alors que celles avec une forte valeur de $m$ seraient à haut risque de développer des métastases. De même, les conséquences des variations du paramètre $\alpha$ sur l'index métastatique à 3 et 5 ans sont présentées dans la Table 3.

\begin{tabular}{|l|l|l|}
\hline \multicolumn{1}{|c|}{$m$} & \multicolumn{1}{c|}{$I M$ à 3 ans } & \multicolumn{1}{c|}{$I M$ à 5 ans } \\
\hline $2.5 \times 10^{-9}$ & 0 & 0 \\
\hline $2.5 \times 10^{-8}$ & 0 & 0 \\
\hline $2.5 \times 10^{-7}$ & 0 & 2 \\
\hline $2.5 \times 10^{-6}$ & 3 & 20 \\
\hline
\end{tabular}

Tab.2 - Variation de l'index métastatique en fonction de $m, 3$ et 5 ans après le diagnostic.

\begin{tabular}{|c|c|c|}
\hline$\alpha$ & $I M$ à 3 ans & $I M$ à 5 ans \\
\hline 0.2 & 0 & 0 \\
\hline 0.48 & 0 & 0 \\
\hline 0.66 & 0 & 4 \\
\hline 0.8 & 3 & 53 \\
\hline
\end{tabular}

Tab.3 - Variation de l'index métastatique en fonction de $\alpha, 3$ et 5 ans après le diagnostic.

\section{3-3 - Variation du paramètre $m$ et simulation du possible impact de la chirurgie sur l’angiogenèse.}

La Table 4 résume les prévisions du modèle 5 ans et 10 ans après chirurgie pour chacune des patientes. Dans les cas des tumeurs composées de plus de $5 \times 10^{10}$ cellules, le modèle fait apparaître que le risque de rechute est très important si aucun traitement complémentaire n'a été donné. D'autre part, nous observons que même dans le cas de la patiente avec une masse tumorale de 1g, l' index métastatique à 10 ans est égal à 1, suggérant l'existence d'un risque faible mais non 
nul de rechute métastatique à 10 ans.

\begin{tabular}{|c|c|c|c|}
\hline Masse tumorale initiale & $\mathrm{n}_{1}=10^{9}$ & $\mathrm{n}_{2}=5 \times 10^{10}$ & $\mathrm{n}_{3}=10^{11}$ \\
\hline IM à 5 ans & 0 & 3 & 5 \\
\hline IM à 10 ans & 1 & 13 & 19 \\
\hline
\end{tabular}

Tab.4 - Evolution de l'index métastatique 5 et 10 ans après exérèse de la tumeur primitive.

\section{3-4 - Variation du paramètre $m$ et chimiothérapie.}

La Table 5 montre la simulation du cas de quatre patientes en situation métastatique qui au début du traitement ont de 1 à 5 métastases détectables. Les calculs donnent le nombre de métastases détectables un an et deux ans après l'arrêt du traitement. On constate une variabilité de la réponse en fonction des valeurs de $m$ : l'efficacité du traitement décroît lorsque $m$ croît. Ainsi la valeur du paramètre $m$ pourrait être prise en compte afin de déterminer, en fonction de chaque cas, le nombre de cycles de chimiothérapie nécessaire à effectuer afin de maintenir l’'IM à 0, 4 à 5 ans après la fin du traitement.

\begin{tabular}{|c|c|c|c|}
\hline$m$ & $\begin{array}{c}I M \text {, au moment du } \\
\text { diagnostic }\end{array}$ & $\begin{array}{c}I M, 1 \text { an après la fin } \\
\text { du traitement }\end{array}$ & $\begin{array}{c}I M, 2 \text { ans après la fin du } \\
\text { traitement }\end{array}$ \\
\hline $2.1 \times 10^{-5}$ & 2 & 0 & 1 \\
\hline $3.8 \times 10^{-5}$ & 3 & 0 & 1.6 \\
\hline $5.4 \times 10^{-5}$ & 4 & 0 & 2.3 \\
\hline $7.6 \times 10^{-5}$ & 6 & 1 & 3 \\
\hline $8.3 \times 10^{-5}$ & 7 & 1.5 & 3.6 \\
\hline
\end{tabular}

Tab.5 - Variabilité de l'efficacité d'une chimiothérapie en fonction des valeurs de $m$, 1 et 2 ans après la fin du traitement $m$.

\section{4 - Discussion}


Le développement d'outils diagnostiques, pronostiques et prédictifs en oncologie clinique connaît un essor constant et doit, à terme, participer à l'établissement d'une médecine personnalisée intégrant les spécificités de chaque patient et de chaque maladie dans la définition d’un traitement individualisé. Les progrès dans le développement et la mise à disposition du clinicien de techniques de plus en plus performantes en imagerie médicale et en génétique (oncogénétique, pharmacogénomique, pharmacogénétique) ont déjà permis d’intégrer, en pratique clinique de routine, des outils éclairant au mieux les équipes soignantes dans le choix d’une stratégie thérapeutique optimale [11]. Les mathématiques appliquées au cancer pourraient également, dans ce contexte, fournir des outils pronostiques de première importance [12], notamment en apportant une information statistique sur un risque de maladie métastatique encore en phase infra-clinique et dont la mise en évidence demeure inaccessible à l'imagerie. Nous avons conçu et développé un modèle mathématique original prédisant l'essaimage métastatique au cours du temps à partir d'une tumeur primitive [4]. Afin de rendre cette modélisation applicable en routine, nous en avons tiré un marqueur, l'index métastatique, susceptible de devenir un outil pronostique pour anticiper la survenue d'une invasion métastatique alors qu'un bilan d'extension est négatif. L'index métastatique que nous présentons ici pourrait caractériser le risque d'évolution métastatique immédiat et secondaire dans le cas de maladie tumorale supposée localisée. L’objectif de ce travail était de présenter cet index et de montrer son intérêt. Nous avons vu que le modèle est apte à discriminer (par le truchement des paramètres $m$ et $\alpha$ ), parmi les patients ne présentant pas de métastases détectables au moment du diagnostic, ceux qui développeront des métastases ultérieurement ; en particulier on pourrait détecter les patientes qui, bien qu'étant dans une classification TNM relativement favorable, ont un grand risque de récidive [14].

Nous avons également vu que le modèle est apte à reproduire l'impact possible de l'acte 
chirurgical sur le risque métastatique, tel que décrit par Peeters et al. [8]. Pour les petites tumeurs, à masse tumorale égale, l'estimation des paramètres $m$ et $\alpha$ semblerait déterminante pour renforcer dans chaque cas la prise de décision de faire ou non une chimiothérapie de couverture, en fonction des valeurs obtenues pour les index métastatiques respectifs. Nos résultats in silico suggèrent fortement que la gradation du risque de maladie métastatique chez les patientes pourrait être précisée par la connaissance des paramètres $m$ et $\alpha$. L'index métastatique pourrait donc constituer un nouvel indicateur de l'agressivité tumorale pouvant compléter ceux déjà existants (statut p53, surexpression Her2/neu, positivité des récepteurs oestrogène,...). Alors qu’il semble exister une large variabilité dans le nombre de cycles de chimiothérapie administré en situation adjuvante $[12,13]$, le calcul de l'index métastatique pourrait orienter le clinicien vers un choix du nombre optimal de cycles de chimiothérapie à effectuer en fonction de la valeur de cet index. En effet, l'intégration de l'effet du traitement anti-cancéreux sur l'index métastatique dans notre modèle permet de décrire l'impact des cures successives de chimiothérapie sur l'évolution des métastases et de calculer le nombre de cycles nécessaires afin de maintenir un index métastatique nul. Afin de proposer un outil utilisable en routine par les cliniciens, la poursuite d'une validation poussée du modèle est nécessaire. Une étude expérimentale non clinique sur petit animal porteur de tumeur orthotopique est actuellement en cours au sein de notre équipe (projet ANR Memorex-PK) afin d'apporter de nouveaux ajustements structurels et numériques au modèle. Il faudra notamment pouvoir estimer les paramètres $a, b, m$ et $\alpha$ à partir d'analyses cliniques courantes effectuées sur la tumeur primitive concernée. Une étude de population permettrait de voir si l'on peut estimer les paramètres $m$ et $\alpha$ chez chaque patient à partir de données anatomo-pathologiques et biologiques (taux de cadhérines, mesure du Ki67.....). Par ailleurs, l'analyse rétrospective de données d'anciens patients atteints de tumeurs solides, 
permettra d’obtenir les éléments nécessaires à la validation définitive de notre modèle. Dans cette optique, nous invitons ainsi les lecteurs du Bulletin du Cancer à nous communiquer toute donnée leur paraissant pertinente afin d'affiner l'adéquation entre les prédictions du modèle et les observations cliniques.

Remerciements : Cette étude a été réalisée grâce au soutien financier de l'Agence Nationale pour la Recherche (ANR - projet Memorex) et de l'Association pour la Recherche contre le Cancer (ARC, subvention n5009). 


\section{Références}

[1] Elias D. Rationnel de la chirurgie oncologique au sein du traitement multimodal des cancers, Bull Cancer 2006; 93 : 775-781.

[2] Spielmann M, Khalil A et André F. Cinétique de prolifération tumorale et efficacité de la chimiothérapie adjuvante. Étude de l'activité mitotique. Cancer du sein, Springer Paris 2006 : 455-463.

[3] Akanuma A. Parameter analysis of Gompertzian function growth model in clinical tumors. Eur. J. Cancer 1978; 14 : 681-688.

[4] Barbolosi D, Benabdallah A, Hubert F, Verga F. Mathematical and numerical analysis for a model of growing metastatic tumors. Math Biosc. 2009; 218:1-14.

[5] Karp G, Bouharmont J, Wissoq JC. Biologie cellulaire et moléculaire. De Boeck, 2004; 850.

[6] Koscielny S, Tubiana M, Le MG. Breast cancer. Relationship between the size of the primary tumour and the probability of metastatic dissemination. Br J Cancer 1984; 49 :709715. 
[7] Peeters C, de Geus LF, Wetphal JR, de Waal RM, Ruiter D, Wobbes T, et al. Decrease in circulating anti-angiogenic factors after emoval of primary colorectal carcinoma coincides with increased metabolic activity of liver metastases. Surgery $2005 ; 137: 246-249$.

[8] Viens P., Roché H., Kerbrat P., Fumoleau P., Guastella JP., Delosier. T. EpirubicinDocetaxel combination in first line chemotherapy for patients with metastatic breast cancer, J. Clin Oncol. 2001;24(4) : 328-335.

[9] Meille C. Modélisation du risque des toxicités induites par des substances thérapeutiques. Université de la Méditerranée : Thèse d’Université, 31 Mars 2009.

[10] Meille C, Iliadis A, Barbolosi D, Frances N, Freyer, G. An interface model for dosage adjustment connects hematotoxicity to pharmacokinetics. Journal of Pharmacokinetics and Pharmacodynamics 2008; 35(6): 619-633.

[11] Coate L, Cuffe S, Horgan A, Hung RJ, Christiani D, Liu G. Germline genetic variation, cancer outcome, and pharmacogenetics. J Clin Oncol. 2010 ; 28:4029-37.

[12] Controversies in design and interpretation of adjuvant clinical trials. Cuzick J. Cancer Invest. 2010;28: 28-34.

[13] Keshtgar M, Davidson T, Pigott K, Falzon M, Jones A. Current status and advances in management of early breast cancer. Int J Surg. 2010; 8:199-202. 
[14] Kronqvist P, Kuopio T, Nykänen H, Anttitinen J, Klemi P. Predicting agressive outcome in T1N0M0 British Journal of Cancer 2004; 91: 277-281. 